\title{
Ethical treatment courses for you!
}

If you're considering branching out into an orthodontic or restorative field, IAS Academy has the course for you. Their aim is to empower dentists to confidently treat their patients with the best and most ethical treatment options available.

The Complete GDP Course runs across two days with the majority of the time including hands-on training and implementation. The modules include everything from patient examination to aesthetic and restorative principles. As well as this, the course incorporates an interactive pre-course webinar, a postcourse assignment, and online mentoring which will aid in your professional development before, during, and after the programme.
You will be provided with the framework to ensure that your patients are treated appropriately and ethically to achieve the best results, as well as 14 hours of CPD and a course provider that offers $24 / 7$ support.

Book your place today.

For more information on upcoming IAS Academy training courses, visit www. iasortho.com or call 01932336470 (press 1).

\section{Free clear aligner webinars in 2022}

Trycare will be hosting FREE 3Dalign webinars with one hour CPD development outcome C throughout 2022.

Delegates will learn how 3Daligners can be integrated into their practices; advantages over other aligner systems; how 3Dalign aim to eliminate the process of 'refinements' which is a problem with most other systems; see examples of advanced case planning; find out about 3Dretain and its advantages for long-term retention.

With some treatment plans taking as little as ten weeks and the majority just ten to 20 aligners, 3Daligners are much quicker, easier, greener and more patient compliant. They enable clinicians to offer patients a simple and cost-effective way to straighten teeth and correct other orthodontic problems.
3Daligner treatment plans include a personalised patient video demonstrating the stages involved and the final outcome.

As the clinician liaises direct with the technicians manufacturing the aligners there is no cumbersome computer interface to negotiate and immediate responses are available. With other systems it can take weeks to receive replacement aligners, during which the patient's treatment can relapse.

With no specialist orthodontic training required it could not be simpler to get started.

For more information about the 3Daligner range, including course details, contact your local Trycare Representative, call 01274885544 or visit www.trycare. co.uk.

\section{Towards simplification in dentistry}

The choice of materials in dentistry is seemingly endless. Patients have become more demanding and regulations have become stricter making running a successful practice a bigger challenge than ever before.

In response to these challenges, for five evenings in November GC Europe brought together dental experts to present a special online congress 'Sharing 100 years of experience towards simplification in dentistry' which attracted over 8,500 delegates from around the world. The congress formed part of the $100^{\text {th }}$ anniversary of the GC Corporation.
With a greater focus on tooth preservation and improved, more durable dental materials, many more patients can be given a healthy smile for a lifetime. The congress offered practical tips, diagnostic tools and straightforward recommendations to give clear guidance to enable practitioners to move forward with confidence to give the very best care to their patients.

This congress, together with all the latest GC webinars, are available to watch on demand at www.gcdentalcampus.com.

For further information contact GC UK on 01908 218999, email info.uk@ gc.dental or visit www.gceurope.com.

\section{To strengthen children's teeth}

Supplied by Trycare in strawberry and melon flavour, BioMin for Kids is available exclusively from dental practices on the high street.

BioMin F for Kids contains tiny bioglass particles made up of fluoro calcium phosphosilicate bioactive glass, which bonds to the teeth and enters the dentinal tubules, where they gradually dissolve over a period of up to 12 hours, slowly releasing calcium, fluoride and phosphate ions. These combine with saliva to form fluorapatite, which is ten times more acid resistant than hydroxyapatite, to strengthen the teeth, aid effective remineralisation of the enamel and prevent fluid flow through the dentinal tubules which triggers sensitivity. Brushing twice daily provides children with up to 24 hours' fluoride protection.

These benefits are also available for older children and adults as BioMin F which has a fresh minty flavour.

BioMin F is a genuine practice builder enabling patients to enhance their smile and improve their oral health and comfort. It is the only toothpaste approved by the Oral Health Foundation for sensitivity relief and remineralisation.

For further information visit the Trycare website, www.trycare.co.uk/biomin, contact your local representative or call 01274885544 .

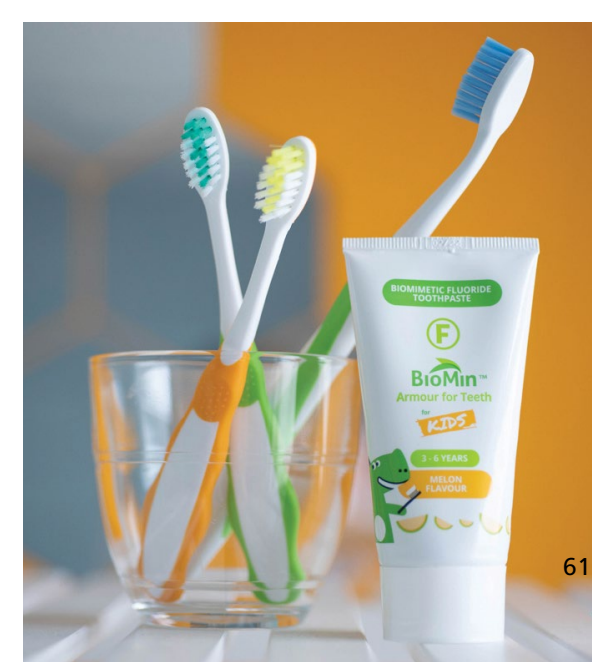

